\title{
Fast performance assessment of mechatronic designs integrating CAD and dynamical models with application on servo actuated designs
}

\author{
Thijs Van Hauwermeiren ${ }^{1,2, \dagger}$, Annelies Coene ${ }^{1,2}$, and Guillaume Crevecoeur ${ }^{1,2}$
}

\begin{abstract}
In this paper an approach is presented that allows to investigate, in a systematic way, how design parameters of a servo actuated system modeled in CAD influence the dynamic behaviour and performance of the system. This is achieved by extracting physical quantities such as mass and inertia in part designs as these are difficult to model analytically when having irregular part shapes. These values are then employed in a symbolic dynamic model to assess the behavior and associated performance through numerical integration. To illustrate the effectiveness of the integrated approach, different slider-crank designs are evaluated for a given motion path. A sensitivity analysis is performed on the basis of gradients extracted from the introduced symbolic dynamical model through algorithmic differentiation. Our approach is flexible and enables precise motion study of actuated mechanisms with calculation times improved by an order of magnitude compared to other methods. In addition, this novel approach depends only on open source software.
\end{abstract}

\section{INTRODUCTION}

Servo actuated systems are key in various machines and industrial applications providing a means for reliable automated motions. Typical goals for such mechatronic systems are accurate tracking of torque, speed and/or positions while maintaining system stability together with the increasing importance of reducing energy consumption [1].

These systems require far-reaching designs that need to integrate mechanical, dynamical and control aspects [2]. Mechanical design of such complex systems is preferably done in Computer Aided Design (CAD) software, with each part drawn individually and the complete system created in an assembly file that defines the relation between parts and imposes the degrees of freedom of the mechanism. These CAD models contain the information about the geometrical properties of parts, such as shape and lengths, as well as physical properties, such as masses and moments of inertia. The set of these properties are the design parameters. These parameters, together with the relations between parts, ultimately define the kinematic and dynamic behavior of servo actuated systems. Correctly identified dynamical models can enable highly accurate position and velocity control of servo systems [3], [4]. The dynamical model can also be leveraged to optimize the design, e.g. see Ref. [5].

There is a clear need to investigate in a systematic way how design parameters of an actuated system that is mechanically designed in CAD, influence the dynamic behavior that can be apprehended by physics-based models. Simulating the

\footnotetext{
${ }^{1}$ Department of Electromechanical, Systems and Metal Engineering, Ghent University, 9000 Ghent, Belgium

${ }^{2}$ EEDT Decision \& Control, Flanders Make

${ }^{\dagger}$ Corresponding author: Thijs.vanhauwermeiren@ugent.be
}

kinematic and dynamic behavior of the complete moving mechanism, referred to as motion study, is an important design step of servo actuated mechanisms and mechatronic systems in general. Using the available info of the motion study, correct operation and adequate strength of chosen components can be ensured. Also, motor selection is usually performed at this stage [6].

A traditional approach is to perform the motion study inside the CAD software used for the mechanical design. It has the obvious advantage that it can directly access all properties defined on the parts and the assembly needed to run the motion study. The CAD software SolidWorks provides a means to perform motion studies [7], but even when using simplified motion laws (e.g. without modeling friction) it executes slowly as will be shown in section IV. This can be explained as the primary focus is on rendering the motion of the mechanism, not on calculating the dynamics of the system. Slow evaluation of the dynamics can hinder comprehensive motion studies and setpoint trajectory optimization [8]. Additionally, motion study software packages embedded into CAD are often only compatible with few other CAD systems and design software, while in general the system designer requires a multitude of software programs for their design to match the high demands on performance and varying requirements (cost, performance, durability, energy efficiency) [1], [9]. Furthermore, these software packages do not allow to control the low-level implementation details of motion study, e.g. numerical integration aspects [10].

This paper present a different approach that allows to integrate both mechanical and dynamical design steps, without being restricted by the chosen $\mathrm{CAD}$ environment for the mechanical design. System parameters from a CAD file are directly extracted into a dynamical physics-based ordinary differential equations based model using an interface script. This approach allows to control all aspects regarding the dynamical model while remaining generally compatible with any CAD software used for the mechanical design.

The dynamical model is implemented as a symbolic function in CasADi [11]. CasADi is an open-source software framework designed for simulating dynamical systems and developed in a toolkit oriented way, providing the building blocks for dynamical analysis and optimal control. Furthermore, it allows to derive gradients in an efficient way using algorithmic differentiation (AD) [12]. Using this functionality a direct link between design parameters and the performance of the system is established, with the additional gain to provide gradient info. Thus, our approach enables sensitivity analysis, guiding the design engineer to favourable 


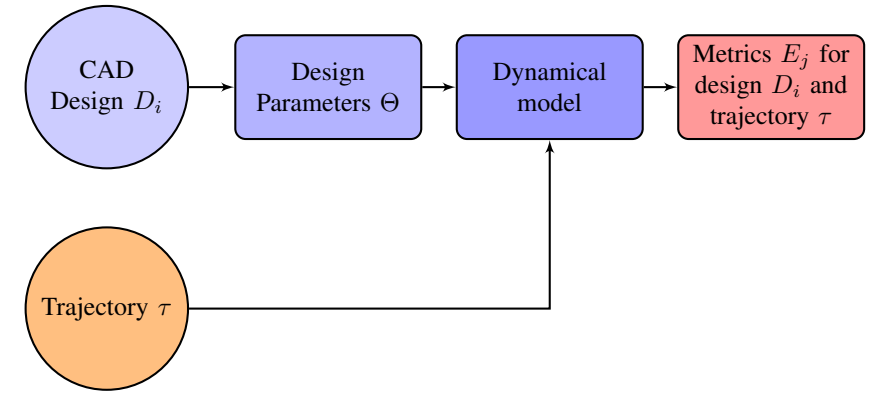

Fig. 1: General overview: Design candidates $D_{i}$ from CAD are represented by design parameters $\Theta$ and are evaluated using a motion trajectory $\tau$ and a dynamics model.

design parameters, and holds the potential to include control aspects into the design methodology. The approach is applied on the case study of a slider-crank servo actuated mechanism.

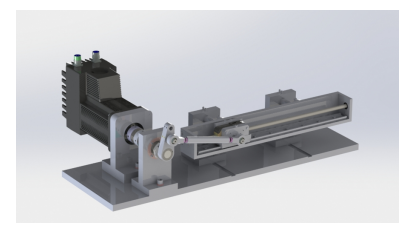

(a) Design with connection rod $L_{2}=150 \mathrm{~mm}$.

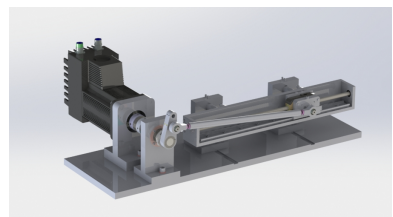

(b) Design with connection rod $L_{2}=350 \mathrm{~mm}$.
Fig. 2: Two design candidates of a slider-crank assembly are shown with different lengths of the connection $\operatorname{rod} L_{2}$, determining the design parameters length, mass and inertia of the conrod.

\section{Methods}

In this section the methods to realize fast performance assessment of mechatronic designs are provided together with implementation details when applied to servo actuated designs.

\section{A. Overview}

The approach, presented in Figure 1, allows for a fast assessment of the performance of different designs $D_{i}$ under application of a predefined motion trajectory $\tau$. The trajectory consist of a list of positions and timings of the driving actuators in the mechanisms. This determines the kinematic behaviour of the complete mechanism. Each aspect of the approach is discussed in detail in the following subsections. Different designs are mechanically modeled in CAD, with an example shown in Figure 2, and exported to a STEP format. From the STEP file, the relevant design parameters $\Theta$ are extracted into the lumped parameter dynamical model. The desired operation of the system is encoded into a trajectory $\tau$. For each design $D_{i}$ and trajectory $\tau$ the performance is expressed in a metric $E_{j}\left(D_{i}, \tau\right)$ that is based on the dynamic behaviour of the complete actuated mechanism.

\section{B. CAD Interface}

The interface script is implemented using the Python API of FreeCAD and is generic in the sense that it can interact with any CAD generated file that is exported to the neutral assembly format STEP AP-203, standardized in ISO 10303-21. STEP files allow for exchange of geometric information of parts and assemblies by means of faces, edges and vertices. FreeCAD [13] is an open-source parametric CAD software featuring a functional graphical user interface (GUI) and deep integration with python.

The scripting ability of FreeCAD was used to implement the interconnection between mechanical and dynamical design step. This scripts allows comparison between different designs of the same assembly or part using the STEP file. In this work, different part designs are modeled in SolidWorks 2019 and the properties of the different designs are compared in FreeCAD using the interface script: mass $M_{F C}$ and inertia $J_{F C}$.

Exchange of material properties with STEP is possible, as the ISO standard has the possibility for inclusion of material properties into the STEP file, yet it is not implemented by the exporter (SolidWorks 2019) nor implemented by the importer (FreeCAD 0.18). The result is that the values of $M_{F C}$ and $J_{F C}$ correspond with solids of density $1000 \frac{\mathrm{kg}}{\mathrm{m}^{3}}$. This issue is resolved by means of a reference design $D_{0}$, of which the actual physical quantities of mass $M_{0}$ and inertia $J_{0}$ in SI units are given by the designer. The other designs are then attributed the correct mass and inertia values by scaling their values with respect to the reference design:

$$
M_{i}=\frac{M_{F C, i}}{M_{F C, 0}} M_{0} \quad \text { (1a) } \quad J_{i}=\frac{J_{F C, i}}{J_{F C, 0}} J_{0}
$$

The moment of inertia depends on the rotation axis and center of rotation. The most straightforward way to allow correct extraction is fixing the origin of the part at the center of rotation before conversion to the STEP format. The CAD files used in the case study in section III together with the interface script and example code have been made available ${ }^{a}$.

\section{Dynamical model}

A method for simulating the multibody dynamics motion study of actuated mechanisms is presented. The system model with state $\mathbf{x} \in \mathbb{R}^{n}$ and dynamics functions $f$ is formalized as:

$$
\dot{\mathbf{x}}=f(\mathbf{x}, \mathcal{U})
$$

With $\mathcal{U}$ some unspecified system input. The dynamical model can be easily modeled in CasADi through the Function class. For ease of working with numerical data, especially for the trajectory (see further below), the dynamics function is discretized with sample time $T_{s}$. The CasADi function calculates the next state $\mathbf{x}(k+1)=f(\mathbf{x}(k), \mathcal{U}(k))$ and can be manipulated to give additional outputs relevant for the performance analysis, such as force, position, velocity and acceleration as these values are calculated as required intermediates for obtaining the next state. The performance metrics are formulated in CasADi on some or all states $\mathbf{x}(k)$ using basic arithmetic, matrix and function operations. As the metrics depends on the dynamics, which in their turn

\footnotetext{
${ }^{a}$ Available at: https://github.com/thvhauwe/cad-interface
} 
depend on the design parameters (and on the trajectory), it is possible to define the CasADi function $E_{j}(\Theta)$ given a fixed trajectory. And because all the arithmetic operations are CasADi functions, it is possible to obtain gradients $\frac{\partial E_{j}}{\partial \Theta_{i}}$ of the performance metric $E_{j}$ w.r.t. a design parameter $\Theta_{i}$ using $\mathrm{AD}$. The advantage of $\mathrm{AD}$ over other techniques such as symbolic or finite differentiation are increased precision and fast evaluation with time and space complexity cost in the same order as evaluating $E_{j}$ itself [12].

\section{Sensitivity analysis}

CasADi allows to express the dynamical model as a symbolic function. In turn, this allows for extraction of the Jacobian $J$ and Hessian $H$ matrix of the performance metrics $E_{j}(\Theta)$ using $\mathrm{AD}$ :

$$
\begin{gathered}
J=\left[\begin{array}{c}
\frac{\partial E_{j}}{\partial \Theta_{1}} \\
\vdots \\
\frac{\partial E_{j}}{\partial \Theta_{n}}
\end{array}\right] \\
H=\left[\begin{array}{ccc}
\frac{\partial^{2} E_{j}}{\left(\partial \Theta_{1}\right)^{2}} & \cdots & \frac{\partial^{2} E_{j}}{\partial \Theta_{1} \partial \Theta_{n}} \\
\vdots & \ddots & \vdots \\
\frac{\partial^{2} E_{j}}{\partial \Theta_{1} \partial \Theta_{n}} & \cdots & \frac{\partial^{2} E_{j}}{\left(\partial \Theta_{n}\right)^{2}}
\end{array}\right]
\end{gathered}
$$

With the Jacobian and Hessian, a second-order Taylor approximation can be constructed at a local design point, which yields valuable information as what design variable influences a certain performance metric the most.

\section{APPLiCATION ON THE SLIDER-CRANK CASE STUDY}

A slider-crank mechanism is a single servo actuated mechanism modeled as a chain with 3 bodies; a crank, connection rod (conrod), with length $L_{1}$ and $L_{2}$ respectively, and a slider. A schematic of the model, depicting all the introduced bodies is shown in Figure 3. The crank shaft is actuated by a servo motor. We consider the crank's angular position $\theta_{1}$ and velocity $\dot{\theta_{1}}$ as system state $\mathbf{x}=\left(\begin{array}{c}\theta_{1} \\ \dot{\theta}_{1}\end{array}\right)$. Writing down the force balance equations (Newton's laws) and moment balance equations in the center of mass of link 1 and 2, and the force balance equations in the slider, we have a linear set of 8 equations resulting in the system transition function. The motor torque $T=\mathcal{U}$ is the system input for the forward dynamic model $f_{d, f}[4]$ :

$$
\ddot{\theta_{1}}=f_{d, f}\left(\theta_{1}, \dot{\theta_{1}}, T\right)
$$

Equivalently, if we consider the desired crank acceleration $\ddot{\theta}_{1}=\mathcal{U}$ as a system input, we can calculate the required motor torque to achieve such motion, yielding the inverse dynamic model $f_{d, i}[4]$ :

$$
T=f_{d, i}\left(\theta_{1}, \dot{\theta_{1}}, \ddot{\theta_{1}}\right)
$$

We model velocity-dependent friction between slider and base, which is the only non-conservative force in the system.

$$
F_{s}=-\mu\left|F_{n}\right| v_{x 3}
$$

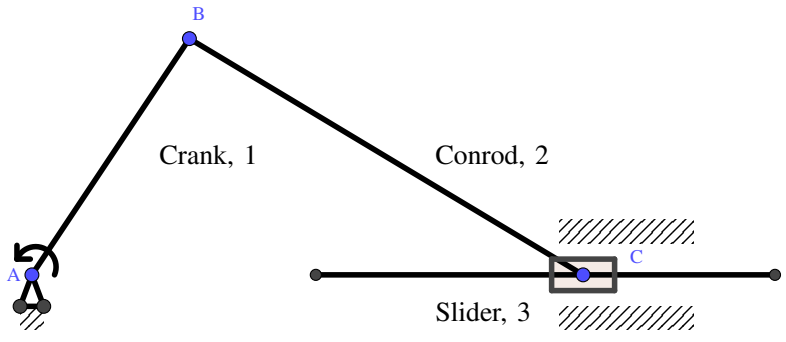

Fig. 3: Simplified representation of a slider-crank. Point A is fixed and only allows rotation of the crank, point $\mathrm{B}$ is free, point $\mathrm{C}$ allows for translation in $\mathrm{x}$-direction.

For performance metrics of the system, we consider the total energy loss $E_{L}$ due to the friction force, equation (7). The root mean square and maximum motor torque, $T_{\text {rms }}$ and $T_{\max }$ respectively, are important motor sizing parameters. Also the maximum occurring forces $F_{i}$ in each of the joints $A, B$ and $C$ need to be considered as system performance metric these are limited by the strength of the material and supporting components such as bearings. In the case of the crank design, additional metrics $E_{\mathrm{L}, \text { norm }}$ and $T_{\text {rms,norm }}$ are introduced which are normalized to the relative distance travelled $X_{\text {dist }}$ because the distance the slider travels is linearly dependent on the length of the crank body $L_{1}$.

$$
E_{L}=\sum_{k}\left|\mu F_{s} v_{x 3}\right|
$$

For each design, we apply the same fixed trapezoidal trajectory $\tau$ for the crank acceleration shown in Figure 5. It consists of a period of constant acceleration, constant speed of $80 \mathrm{rpm}$, constant deceleration. This is a common trajectory in industrial servo applications [14]. The trajectory of 4 seconds is discretized using a sample time of $T_{s}=5 \cdot 10^{-4} \mathrm{~s}$. In total, the trajectory consists out of 8000 time steps. Using the inverse dynamic model we obtain the (required) motor torque, position, velocity, acceleration and force component in each body at each discrete time instance.

\section{Results}

\section{A. Motion study of parametrized conrod}

Five different designs of the conrod with varying lengths $L_{2}$ are investigated that also influence the corresponding mass $M_{2}$ and inertia $J_{2}$ of the body. A render of the CAD model of this setup is shown in Figure 2. The relative center of mass is fixed at 0.5 , because the conrod is modeled as a cilinder. In this study the design parameters are $\Theta=$ $\left[L_{2}, M_{2}, J_{2}\right]$. The extracted parameters are reported in Table I, scaled relative to the values of the base design $D_{0}$ using equation (1). The physical quantities from the base design are given in Table II.

The results of the motion study are presented in the table using the previously defined performance metrics, Table III. 


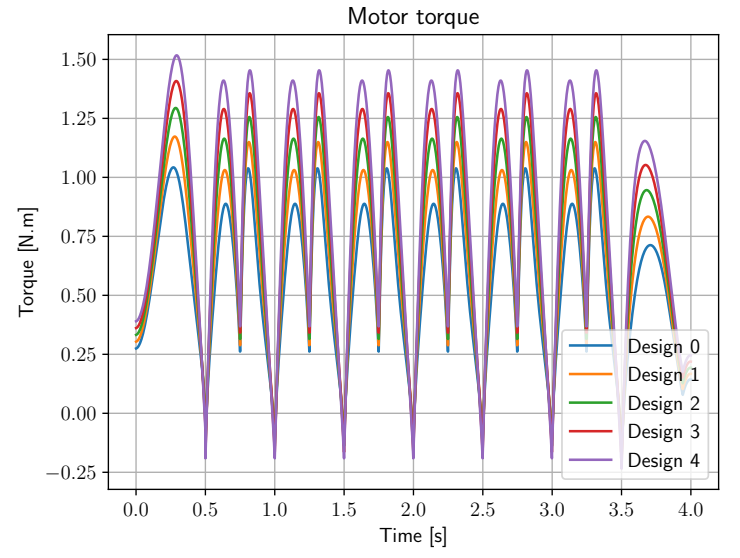

(a)

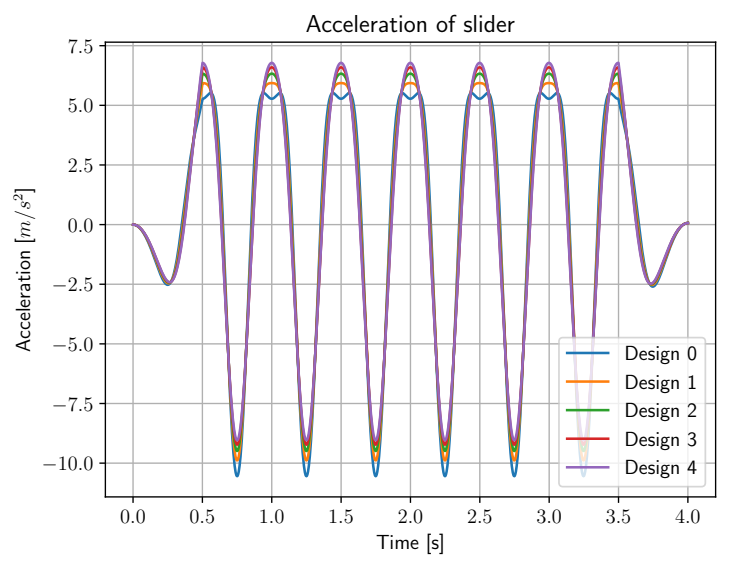

(b)

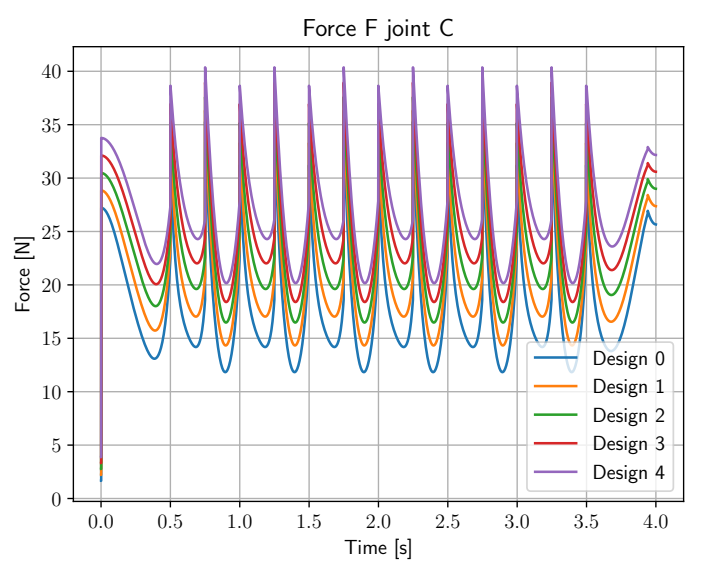

(c)

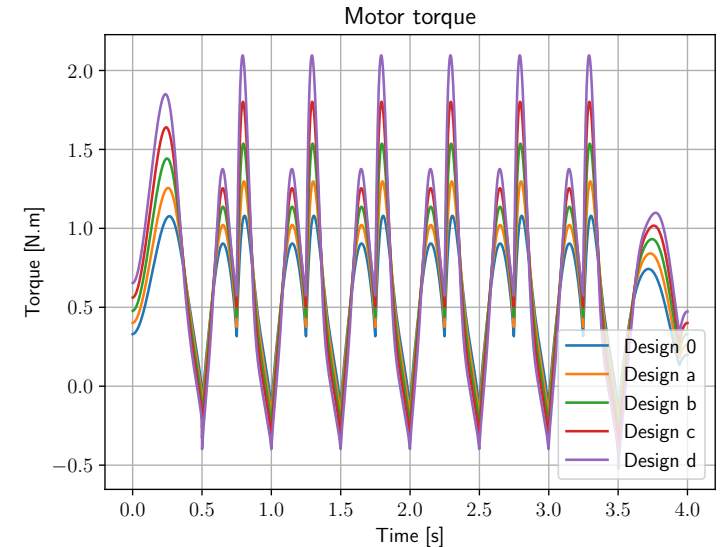

(d)

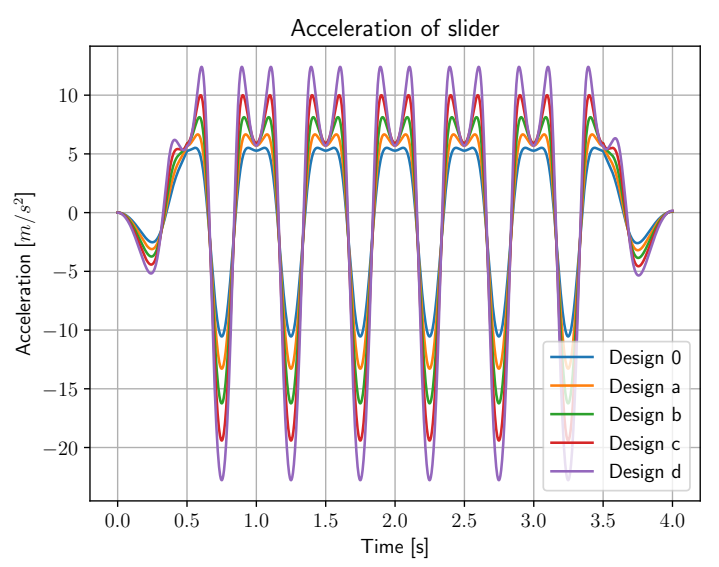

(e)

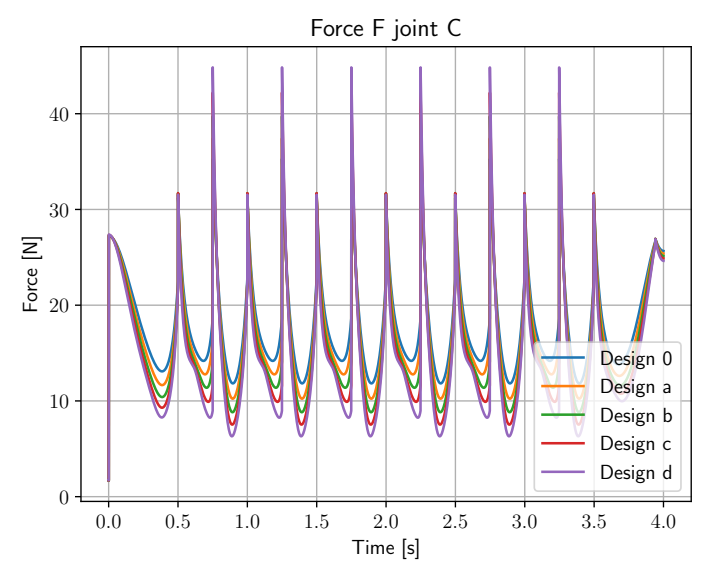

(f)

Fig. 4: Simulation results. Left column (Figures (a) - (c)) are the results for the conrod motion study, right column (Figures (d) - (f)) for the crank motion study. 


\begin{tabular}{ll|ccc}
\hline Design & $L_{2}[\mathrm{~mm}]$ & $L_{2}[-]$ & $M_{2}[-]$ & $J_{2, \mathrm{x}}[-]$ \\
\hline$D_{0}$ & 150 & 1.00 & 1.00 & 1.00 \\
$D_{1}$ & 200 & 1.33 & 1.33 & 2.36 \\
$D_{2}$ & 250 & 1.67 & 1.67 & 4.59 \\
$D_{3}$ & 300 & 2.00 & 2.00 & 7.91 \\
$D_{4}$ & 350 & 2.33 & 2.33 & 12.55 \\
\hline
\end{tabular}

TABLE I: Design candidates of the conrod motion study with parameters length, mass and inertia (three last columns) relative to design $D_{0}$.

The plots of the motor torque, acceleration of the slider and force magnitude on the slider joint $\mathrm{C}$ are shown in Figure 4. Other physical quantities such as velocity and acceleration are not shown for brevity.

From the data in Table III, it is clear that the design $D_{0}$, with the smallest conrod length $L_{2}$ has the best energy performance and requires the least amount of motor torque to drive the system. This is supported by the force on the slider, depicted in Figure 4c, the short conrod design has a smaller force acting upon the slider.

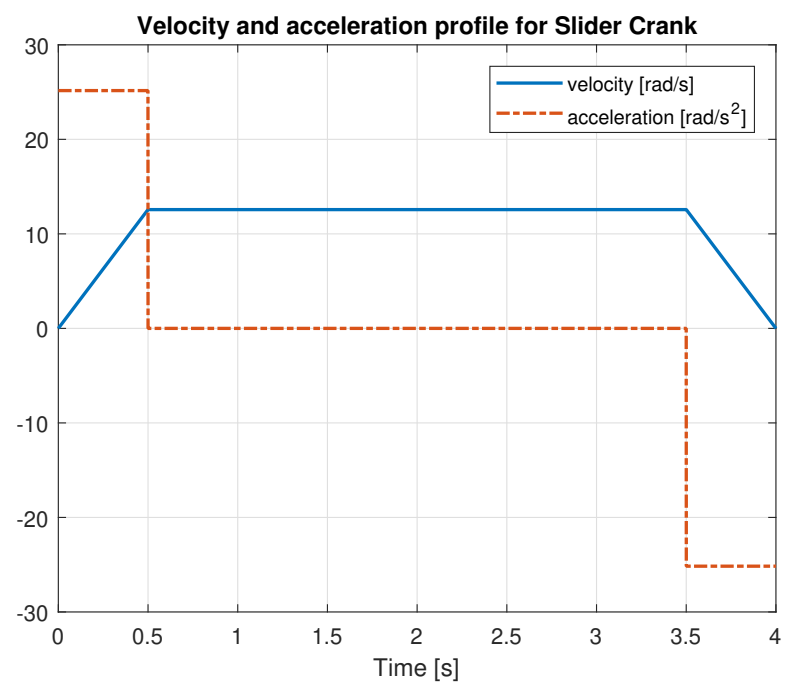

Fig. 5: Fixed trapezoidal trajectory for each design. The goal velocity is $80 \mathrm{rpm}(12,73[\mathrm{rad} / \mathrm{s}])$. Velocity is $\dot{\theta_{1}}$, acceleration is $\ddot{\theta_{1}}$.

\section{B. Motion study of parametrized crank}

In the study of the crank design, center of mass $C_{M 1}$ is also a parameter that changes with crank length $L_{1}$. The design parameters of that study are represented by $\Theta=\left[L_{1}, M_{1}, J_{1}, C_{M 1}\right]$. The longer the crank, the longer the distance the slider travels for the given motion trajectory. Results are reported in Table V.

The shortest crank design $D_{0}$ has the lowest energy consumption $E_{L}$. When normalizing for the distance travelled, $E_{\mathrm{L}, \text { norm }}$, a longer crank yields a more efficient mechanism. However, when studying the acceleration profile Figure 4e, a longer crank substantially distorts the sinusoidal motion profile. This can be explained by the ratio $\frac{L_{2}}{L_{1}}$, when the ratio is $\infty$, the slider will have a perfect sinusoidal motion at constant crank speed.

\section{Sensitivity Analysis}

Using the designs 1 to 5 from the conrod motion study, second-order Taylor approximations are made for $E_{L}\left(J_{2}\right)$, $E_{L}\left(M_{2}\right)$ and $E_{L}\left(L_{2}\right)$ and presented in Figure 6. Clearly, for design $D_{0}$ the length $L_{2}$ is the dominant parameter for $E_{\mathrm{L}}$, whereas for $D_{5}$ both mass and length of the conrod body have approximate equal influence for the energy efficiency of the motion. The inertia has no impact for energy efficiency in this specific servo actuated design study.

\section{Benchmark}

Simulating all the positions, velocities, accelerations, forces with the dynamical model and calculating all the performance metrics of the crank motion study for the 8000 time step trajectory had a mean execution time of $85.6 \mathrm{~ms}$ (maximum: $87 \mathrm{~ms}$, minimum $85.1 \mathrm{~ms}$, standard deviation $0.4 \mathrm{~ms}, 100$ samples used). As a comparison, the same trajectory was run in SolidWorks Motion Analysis tool. Rendering is disabled and all other options left at default to allow fair comparison. Using the same fixed sample time $T_{s}=5 \cdot 10^{-4}$, SolidWorks took a considerable longer time to calculate the same motion profile: $2419,4 \mathrm{~ms}$ (maximum: $2700 \mathrm{~ms}$, minimum $2330 \mathrm{~ms}$, standard deviation $101,7 \mathrm{~ms}$ ). It is fair to mention that in SolidWorks a more complex CAD model is present, whereas the model in CasADi is a 1D, reduced dynamical model. More importantly however is that there is no apparent way to export the values or quantities from SolidWorks so that they can be used to calculate custom-defined performance metrics. Equally, the integrator algorithms are provided by SolidWorks, with only limited configurability, and are restricted to execute on CAD models defined within SolidWorks.

\section{E. Summary of results}

Two motion studies were carried out in this section. Different designs of the connection rod and crank of the slider-crank mechanism were evaluated using the presented approach. It was demonstrated how different designs were ranked based on predefined performance criteria such as energy loss. In the sensitivity analysis was shown how the symbolic dynamical model can be leveraged to gain insight in which physical quantity has the most impact on the performance criteria, in the case of the slider-crank the physical length of the conrod was the decisive parameter for energy efficiency.

\section{CONCLUSion}

A novel approach is presented to evaluate the performance of mechatronic designs, integrating CAD and motion study. The developed method is able to simulate the complete dynamic behaviour of a complex servo actuated design over thousands of time steps in under 100 milliseconds calculation time, at least one order of magnitude faster than the compared commercially available tool. Our method offers the 


\begin{tabular}{cccccccc}
$M_{1}[\mathrm{~kg}]$ & $M_{2}[\mathrm{~kg}]$ & $M_{3}[\mathrm{~kg}]$ & $L_{1}[\mathrm{~m}]$ & $L_{2}[\mathrm{~m}]$ & $\mu[-]$ & $J_{1}\left[10^{-3} \mathrm{kgm}^{2}\right]$ & $J_{2}\left[10^{-3} \mathrm{kgm}^{2}\right]$ \\
\hline 0.223 & 0.348 & 0.795 & 0.05 & 0.15 & 2.8715 & 0.6186 & 0.9411
\end{tabular}

TABLE II: Physical quantities of all design parameters for $D_{0}$.

\begin{tabular}{c|cccccc}
\hline Design & $E_{\mathrm{L}}$ & $T_{\mathrm{rms}}$ & $T_{\max }$ & $F_{\mathrm{A}, \max }$ & $F_{\mathrm{B}, \max }$ & $F_{\mathrm{C}, \max }$ \\
\hline$D_{0}$ & 1.00 & 1.00 & 1.00 & 1.00 & 1.00 & 1.00 \\
$D_{1}$ & 1.19 & 1.15 & 1.13 & 1.05 & 1.05 & 1.03 \\
$D_{2}$ & 1.35 & 1.28 & 1.24 & 1.10 & 1.10 & 1.07 \\
$D_{3}$ & 1.49 & 1.41 & 1.35 & 1.16 & 1.16 & 1.10 \\
$D_{4}$ & 1.63 & 1.52 & 1.46 & 1.21 & 1.22 & 1.14 \\
\hline
\end{tabular}

TABLE III: Performance metrics of the conrod design motion study, values relative to design $D_{0}$.

\begin{tabular}{cc|cccc}
\hline Designs & $L_{1}[\mathrm{~mm}]$ & $L_{1}[-]$ & $M_{1}[-]$ & $C_{M 1}[-]$ & $J_{1, \mathrm{z}}[-]$ \\
\hline$D_{0}$ & 50 & 1.0 & 1.00 & 0.79 & 1.00 \\
$D_{a}$ & 60 & 1.2 & 1.14 & 0.74 & 1.38 \\
$D_{b}$ & 70 & 1.4 & 1.29 & 0.70 & 1.85 \\
$D_{c}$ & 80 & 1.6 & 1.43 & 0.67 & 2.41 \\
$D_{d}$ & 90 & 1.8 & 1.58 & 0.64 & 3.08 \\
\hline
\end{tabular}

TABLE IV: Design candidates of the crank motion study with parameters length, mass and inertia relative to design $D_{0}$.

capability to deliver valuable insights to the human designer, whom is able to assess what small changes in design can impact the operation and performance of the system.

Future work could leverage the fast calculation time and gradient info to enable gradient-based design optimization routines suited specifically for servo actuated mechatronic designs. Furthermore, it is possible with the presented approach to not only parameterize the design but also the trajectory to optimize the motion of the mechanism.

Finally, a straightforward interface has been provided to perform precise motion study in a flexible manner irrespective of the used CAD software to model the mechanical design.

\begin{tabular}{c|cccc}
\hline Design & $E_{\mathrm{L}}$ & $T_{\mathrm{rms}}$ & $T_{\max }$ & $E_{\mathrm{L}, \text { norm }}$ \\
\hline$D_{0}$ & 1.00 & 1.00 & 1.00 & 1.00 \\
$D_{a}$ & 1.10 & 1.20 & 1.13 & 0.95 \\
$D_{b}$ & 1.18 & 1.40 & 1.26 & 0.91 \\
$D_{c}$ & 1.24 & 1.60 & 1.39 & 0.88 \\
$D_{d}$ & 1.28 & 1.80 & 1.52 & 0.86 \\
\hline & & & & \\
\hline Design & $T_{\mathrm{rms}, \text { norm }}$ & $F_{\mathrm{A}, \max }$ & $F_{\mathrm{B}, \max }$ & $F_{\mathrm{C}, \max }$ \\
\hline$D_{0}$ & 1.00 & 1.00 & 1.00 & 1.00 \\
$D_{a}$ & 0.94 & 1.09 & 1.07 & 1.06 \\
$D_{b}$ & 0.90 & 1.18 & 1.15 & 1.13 \\
$D_{c}$ & 0.87 & 1.28 & 1.24 & 1.20 \\
$D_{d}$ & 0.85 & 1.39 & 1.33 & 1.27 \\
\hline
\end{tabular}

TABLE V: Performance metrics of the crank design motion study, values relative to design $D_{0}$.

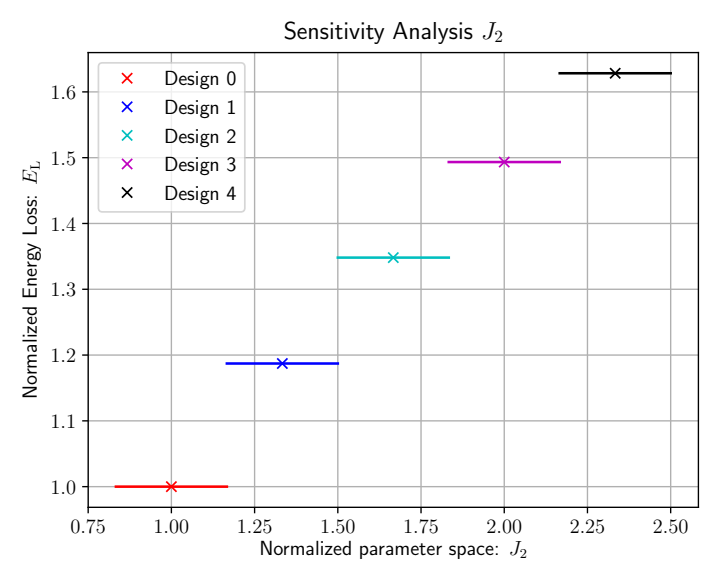

(a)

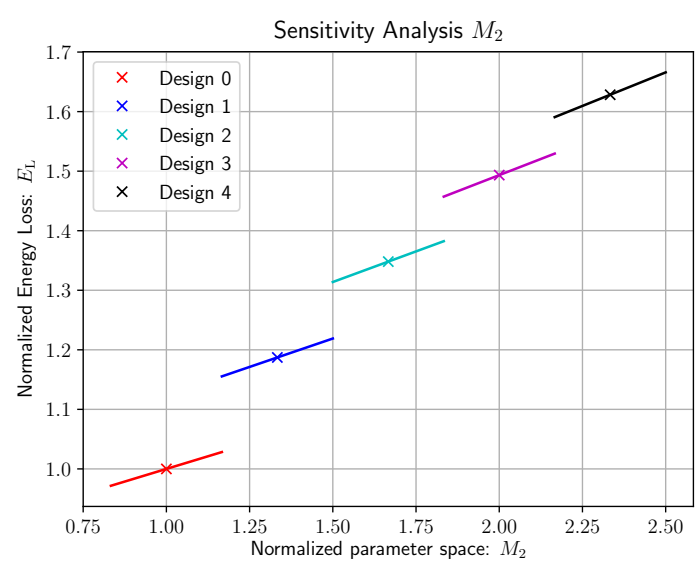

(b)

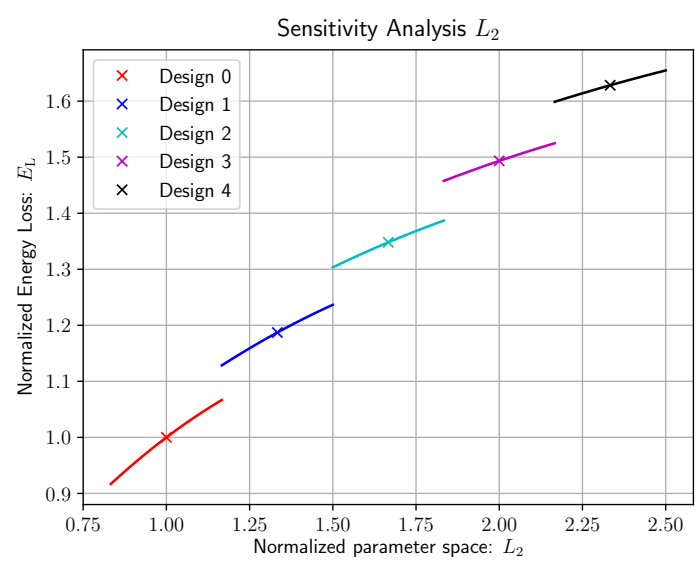

(c)

Fig. 6: Sensitivity Anaylsis of the conrod designs showing a second order taylor expansion at the five different designs. 


\section{ACKNOWLEDGEMENT}

This work was financially supported by the Flanders Make project 'Product and Assembly co-design'. A.C. is supported by the Research Foundation - Flanders (FWO) through a postdoctoral fellowship. This research received partial funding from the Flemish Government under the "Onderzoeksprogramma Artificiële Intelligentie (AI) Vlaanderen” programme.

\section{REFERENCES}

[1] Giovanni Berselli, Federico Balugani, Marcello Pellicciari, and Michele Gadaleta. Energy-optimal motions for Servo-Systems: A comparison of spline interpolants and performance indexes using a CADbased approach. Robotics and Computer-Integrated Manufacturing, 40:55-65, 2016.

[2] James T. Allison. Plant-Limited Co-Design of an Energy-Efficient Counterbalanced Robotic Manipulator. Journal of Mechanical Design, 135(10), 08 2013. 101003.

[3] Sungmin Kim. Moment of Inertia and Friction Torque Coefficient Identification in a Servo Drive System. IEEE Transactions on Industrial Electronics, 66(1):60-70, 2019.

[4] Tom Lefebvre, Frederik De Belie, Frank Naets, and Guillaume Crevecoeur. International Conference on Advanced Intelligent Mechatronics Adaptive Approximate Dynamic Optimization : a slider-crank case study. 2019 IEEE/ASME International Conference on Advanced Intelligent Mechatronics (AIM), pages 1509-1515, 2019.

[5] Huilong Yu, Federico Cheli, and Francesco Castelli-Dezza. Optimal design and control of 4-IWD electric vehicles based on a 14 DOF vehicle model. IEEE Transactions on Vehicular Technology, 67(11):10457-10469, 2018.

[6] H. J. van de Straete, P. Degezelle, J. De Schutter, and R. J. M. Belmans. Servo motor selection criterion for mechatronic applications. IEEE/ASME Transactions on Mechatronics, 3(1):43-50, March 1998.

[7] S. Mary. White paper: Understanding Motion Simulation. Technical report.

[8] Nick Van Oosterwyck, Foeke Vanbecelaere, Michiel Haemers, David Ceulemans, Kurt Stockman, and Stijn Derammelaere. CAD Enabled Trajectory optimization and Accurate Motion Control for Repetitive Tasks. IEEE International Conference on Control and Automation, ICCA, 2019-July:387-392, 2019.

[9] Tobias Larsson. Multibody dynamic simulation in product development. (May), 2001

[10] David Griffiths and Desmond J. Higham. Numerical Methods for Ordinary Differential Equations, Second Edition. 2008.

[11] Joel A.E. Andersson, Joris Gillis, Greg Horn, James B. Rawlings, and Moritz Diehl. CasADi: a software framework for nonlinear optimization and optimal control. Mathematical Programming Computation, 11(1):1-36, 2019.

[12] Assefaw H. Gebremedhin and Andrea Walther. An introduction to algorithmic differentiation. Wiley Interdisciplinary Reviews: Data Mining and Knowledge Discovery, 10(1):1-21, 2020.

[13] J. Riegel, W. Mayer, and Y. v. Havre. FreeCAD [Software] Available from http://www.freecadweb.org, Version 0.18.

[14] Dario Richiedei and Alberto Trevisani. Analytical computation of the energy-efficient optimal planning in rest-to-rest motion of constant inertia systems. Mechatronics, 39:147-159, 2016. 